\title{
Smart Broadcast Technique for Improved Video Applications over Constrained Networks
}

\author{
U. Ukommi \\ Faculty of Engineering and Physical Sciences \\ University of Surrey \\ Guildford, Surrey, United Kingdom.
}

\begin{abstract}
-improved wireless video communication is challenging since video stream is vulnerable to channel distortions. Hence, the need to investigate efficient scheme for improved video communications. This research work investigated broadcast schemes, and proposes smart broadcast technique as a solution for improved video quality over constrained network such as wireless network under tight constraints. The scheme exploits the concept of video analysis and adaptation principles in the optimization process. The experimental results obtained under different channel conditions demonstrate the capability of the proposed scheme in terms of improving the average received video quality performance over a constrained network.
\end{abstract}

Keywords-Video communication; broadcast; video quality; wireless network

\section{INTRODUCTION}

Video communication over a wireless channel is more error-prone compared to that of a wired channel due to varying channel conditions and resource constraints. Demand for improved video services is rapidly growing in the society. A typical video communication system consists of video source, source encoder, channel encoder and receiving terminal for reception and display of the transmitted video signal. The video encoder such as H.264/AVC [1] performs video compression and support error resilience features [2] [3]. Wireless technology such as Mobile WiMAX provide delivery channel for video applications over wireless paltform. Mobile WiMAX uses Orthogonal Frequency Division Multiple Access (OFDMA) [4], which divides the available resources into a number of subchannels [5]. Modulation schemes such as Quadrature Phase Shift Keying (QPSK) and Quadrature Amplitude Modulation (QAM) are also supported in OFDMA with variable channel coding rates [6] [7] which offer channel protection for improved video communications. Factors such as Signal-to-Noise Ratio (SNR) determine the performance of a wireless transmission [8]. SNR takes the relative factors such as path loss, cable loss into account [9].

Wireless video communication is becoming indispensable means of communication in the society due to the mobility, flexibility and portability. It is capable of satisfying client demand anywhere at anytime. However, there are many challenges in wireless video communication channels such as resource constraints, varying network characteristics. These factors influence the quality performance of video services over challenging networks. Hence, this research report focuses on efficient scheme, utilizing content characterization to maximize the received video quality performance under constrained networks.

\section{RELATED WORK}

Various schemes for video transmission have been discussed in the literature including Unequal Power Allocation for Scalable Video Transmission, where base layers of scalable video are allocated more error protection level compared to the enhancement layer [10]. An Unequal Error Protection Scheme for Object-based Video Communications, where different quantization parameters are allocated for coding of each object in the video sequence [11]. Selforganized Content and Routing in Intelligent Broadcast Environments (SCRIBE) is presented in [12] with the objective of minimizing the redundancy associated with convectional flooding broadcast scheme. SCRIBE achieves its objective by adaptively controlling the message path. Intelligent Broadcast System for Enhanced Personalizedservices based on contents semantic is discussed in [13] where viewers satisfaction is increased by serving user preferences. In [14] multimedia adaptation is adopted in which based on the fact viewers are more interested in a certain region or clip. Based on this fact, different adaptation schemes have been proposed [15] [16]. These schemes aim to reduce the quality for regions that are little or no interest for the user and to increase the quality on the Region of Interest (ROI). The idea is based on human visual system that has different sensitivity to different visual areas [17]. In addition to the existing technologies, improving the quality of video applications over constrained network based on the characterization of video stream is proposed in the Smart Broadcast Technique (SBT). The rest of the paper is organized as follows. Section III describes the proposed scheme. Section IV presents the system architecture scheme. Section V describes the simulation methodology. Section VI presents the results and discussion of the research. Finally, the paper is concluded in Section VII with future work.

\section{The PRoposed TECHNIQUE}

The proposed Smart Broadcast Technique (SBT) for improved video broadcast applications is based on the dynamic nature of video content characterization. The main objective of SBT is to enhance transmission strategy in order to improve the received video performance over constrained network. In the scheme, video transmission parameters are adapted according to the characteristic of the video stream. The characterization process includes classification and 
prioritization of video streams based on their respective properties. Such property include content characteristic which is employed as an index in the adaptation process. The scheme is designed to analyze video streams with relative motion characteristic and improves its protection level against impact of channel errors. The proposed scheme aims at maximizing the average received video performance through intelligent adaptation of the limited transmission resources.

In the characterization process, optical flow algorithm of Lucas and Kanade [18] is used in the research to analyze video characteristics. Video contents are analyzed and the average motion activity in video scene is estimated. The motion activity estimation process is carried out by identification of significant feature points in the video scene. Feature point in this concept is defined as a point of the object in video scene that can be easily detected and tracked from frame to frame. Types of image features points include edges, blobs, ridges and corners [19]. In the measurement process, the object is first detected and analyzed to determine the changes in motion activity. Feature point relative difference in terms of pixel displacement between successive video frames quantifies motion vector of the feature point.

A typical video scene consists of objects characterized by spatial characteristics (number and shape of objects) and temporal characteristics. Changes in the motion characteristics between successive video scenes are mainly caused by object motion and global motion [20]. In the estimation of motion activity level of a video scene, the total average motion activity over a given video sequence is normalized by the spatial and temporal resolutions to maintain consistency across different video sequences. The mathematical model for the estimation process is given by [21]: $\mathrm{M}_{\mathrm{s}}=\sum_{i=0}^{L-1} \Delta_{F} \times \frac{R}{T}$, where $M_{S}$ represents the motion activity level of the video sequence over $L$ number video frames. $\Delta_{F}$ is the motion activity level of a video frame. $R$ and $T$ are the spatial and temporal resolutions of the video sequence, respectively.

\section{SYSTEM ARCHITECTURE}

The proposed SBT system architecture consists of the video source, channel and the receiving components as shown in Figure 1. Video communications process includes capturing, content analysis, encoding, adaptation and transmission. The receiving section consists of receiver, decoder and display unit. Video broadcasting involves capturing of natural scene by video camera, encoding by video compression algorithms and transmission of compressed video streams over a communication channel. The encoding block performs video compression function by exploiting redundancies in video sequence and application of various algorithms to enhance robustness of video streams.

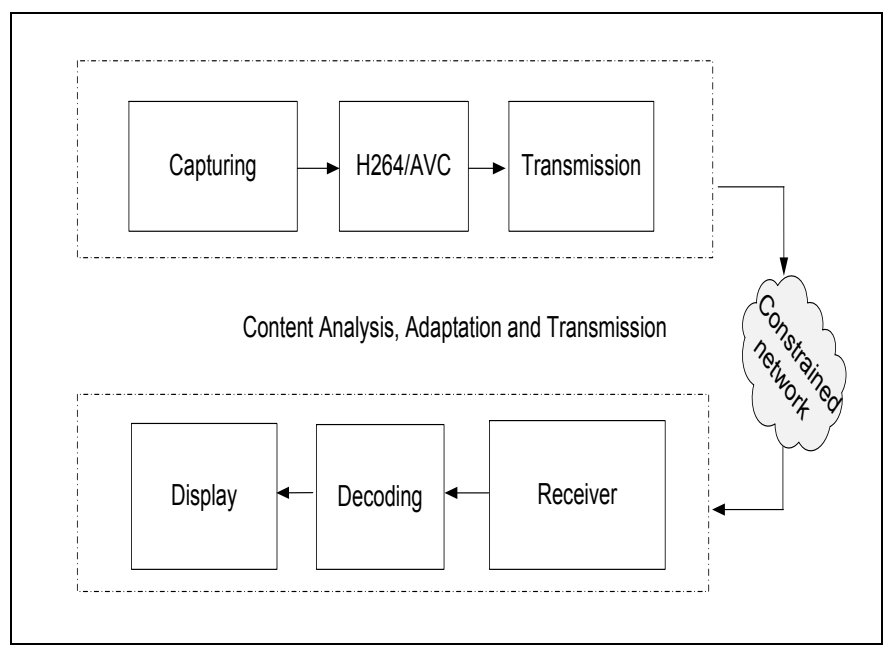

Fig. 1. SBT System Architecture

Wireless video distribution is more challenging due to varying channel characteristic and resource constraints and high bit error rates which affect the received video quality performance. In the system design, the compressed video streams are transmitted through the constrained network. At the receiving section, the transmitted video stream is decoded frame by frame using H.264/AVC codec. Finally, the reconstructed video stream is processed and displayed on the receiving device. More details on the video communication systems including digital video compression, transmission and decoding are discussed in the literature [22].

In many wireless video communication scenarios, the primary constraint for reliable communication with good video quality performance arises from resource constraints [23]. Video applications and services require adequate transmission resources to attain good quality. However, it becomes challenging under constrained network with limited transmission resources to support improved received video quality [24]. The proposed scheme aims at maximizing the average received video quality performance for a set of video applications over a constrained network as illustrated in Figure 1. The total video distortions consist of source distortion and channel distortion [25] [26]. In the analysis, using a preencoded video stream the received video quality performance depends on the distortion of the transmitted media streams due to channel errors. Hence, the scheme looks into more efficient strategy that maximizes the usage of the limited network resources to minimize the channel distortion and improve the received video quality. Thus, maximize the average received video broadcast services within a tight constrained budget. However, in order to devise an efficient broadcast system, the research investigates the impact of channel errors and resource constraints on received video quality performance. 


\section{SimUlation}

The simulation process is performed using different standard test video sequences representing different content characterization, ranging from high motion characteristic (HP), to low motion content characteristic (LP). In the classification process, test video sequences are clustered into group based on the similarity of content characteristics, temporal and spatial resolution characterization.

H.264/AVC reference software, version 15.1 (JM 15.1) [27] is employed in pre-encoding of the test video sequences. The scheme is tested with standard sample test video sequences: Soccer, Foreman, Weather and Akiyo, all in Common Intermediate Format (CIF). The test video sequences are encoded at average bitrates of $0.768 \mathrm{Mbps}$. The frame rate is fixed at 30 frames per second, and the Group of Picture (GOP) size of 8 was employed in the compression process for all the test video sequence. In each of the test video sequence, a total number of 300 frames were processed in IPPP... format (first frame of each sequence is intra-coded, followed by Pframes). Content Adaptive Binary Arithmetic Coding (CABAC) technique is adopted in the compression. The compressed video streams are segmented into slice. The slice [28] is encapsulated in RTP/UDP/IP [29] for transmission through the protocol stack of simulated broadcast system. At the Network Abstraction Layer (NAL), slice output of the VCL is placed in NALU [30] prior to transmission. For optimization of the payload header [31] and reduction of high loss probability the NALU length is fixed at 512 bytes for the test video sequences. RTP transport is augmented by a control protocol for monitoring of the data delivery and provision of feedback on the reception quality [32]. The compressed video streams are then transmitted through simulated wireless Chanel.

The channel conditions are simulated with error traces [33], generated from simulated wireless channel conditions for 16QAM, 1/2 Modulation and Coding Scheme (MCS), with a range of SNR levels. The error patterns are obtained by comparing the data bits within the original data slot to the transmitted data slot. If there is any bit error within the data slot, it is then declared as an error. The error traces with the similar SNR are used to corrupt the compressed video streams transmitted through the simulator. The SNR levels for the video streams are distributed using lookup tables. The look-up tables translate the bit error rate information into distortion levels, which are used in the distribution of the transmission resources among the video streams. The transmission parameters of the video streams are adjusted based on the content characterization of the compressed video streams. The resource distribution is carried out initially by equal allocation of transmit resources for all media streams, and then incremented based on the content characterization of the video streams. The uniqueness of the scheme can be recognized in terms of simplicity of the system model and resource allocation strategy. The experimental results were averaged over ten simulations carried out repeatedly in order to obtain stable results and evaluate the performance of the proposed scheme.
At the receiving section, the transmitted video streams are demodulated and decoded using H.264/AVC reference software version 15.1 (JM 15.1). The error concealment with frame copy mode is employed for concealment of corrupted video packets. When a packet is lost, the RTP sequence number enables the decoder to identify the lost packet such that the location of the corrupted packet in a frame is identified and concealed. Peak Signal-to-Noise Ratio (PSNR) [34] is used in estimating the received video quality performance.

As a measure of the objective function which can be defined as maximizing the average received video quality performance among a set of video streams over a constrained network, PSNR [35] is employed to measure the performance of the proposed system in terms of the average received video quality of the transmitted video streams. PSNR metric is widely employed in the field of video quality performance measurement, though do not have strong correlation with subjective experiment [36]. Table 1 presents the simulation conditions and input parameters.

TABLE I. PARAMETERS For SimULATIONS

\begin{tabular}{|l|l|}
\hline System & Parameter \\
\hline Test video sequence & Soccer, Foreman, Weather, Akiyo \\
\hline Source encoder & H.264/AVC reference software \\
\hline Frames format & IPPP... \\
\hline Spatial resolution & CIF $(352 * 288)$ \\
\hline GOP & 8.0 \\
\hline Frame rate & $30 \mathrm{fps}$ \\
\hline Packet size & 512 bytes \\
\hline Channel Coding & CTC \\
\hline Permutation scheme & PUSC \\
\hline Path loss model & ITU-R \\
\hline Quality Measurement & PSNR \\
\hline
\end{tabular}

\section{RESUlt AND Discussion}

The average received video quality performances of tested video sequences are measured using PSNR algorithm. The simulations were carried out to assess the performance of the proposed scheme in terms of average received video quality performance over constrained network. Readings of the PSNR values were taken from the reconstructed video frames by comparing with the original video frames. The PSNR values represent the received video quality performance. The higher the PSNR values the better the received video quality performance. From Table 2, the video quality performance across the tested video sequences varies according to the video content characterization. The average received video quality performance of video content characterized with high motion (HP) recorded improvement when transmitted on SBT scheme. The enhancement in the received video quality performance is due to the fact that the proposed SBT system improves the protection level of the video streams with high motion characterization by improving the SNR level. The enhancement in the protection level mitigates the impact of channel errors on the reconstructed video quality. 
Table 2 shows the comparative performance of the received video quality "With SBT" scheme and "Without SBT".

TABLE II. COMPARATIVE RECEIVED VIDEO QUALITY PERFORMANCE "WITH SBT" SCHEME AND "WITHOUT SBT" SCHEME.

\begin{tabular}{|c|c|c|c|c|c|c|}
\hline \multirow[t]{2}{*}{ 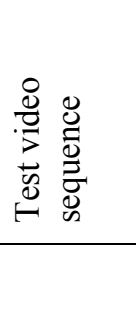 } & \multirow[t]{2}{*}{ 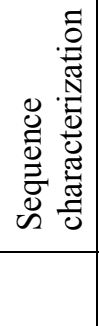 } & \multirow{2}{*}{ 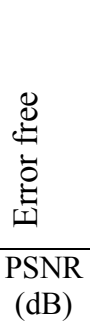 } & \multicolumn{2}{|l|}{ 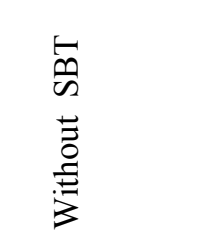 } & \multicolumn{2}{|l|}{ 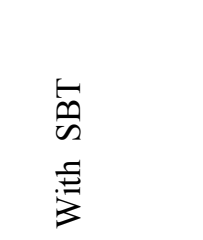 } \\
\hline & & & $\begin{array}{l}\text { SNR } \\
(\mathrm{dB})\end{array}$ & $\begin{array}{c}\text { PSNR } \\
\text { (dB) }\end{array}$ & $\begin{array}{l}\text { SNR } \\
(\mathrm{dB})\end{array}$ & $\begin{array}{c}\text { PSNR } \\
(\mathrm{dB})\end{array}$ \\
\hline Soccer & $\mathrm{HP}$ & 36.60 & 11.60 & 33.52 & 12.15 & 35.23 \\
\hline Foreman & $\mathrm{HP}$ & 38.32 & 11.60 & 35.47 & 12.15 & 36.05 \\
\hline Weather & LP & 43.99 & 11.60 & 40.03 & 11.05 & 36.66 \\
\hline Akiyo & LP & 51.08 & 11.60 & 50.16 & 11.05 & 44.66 \\
\hline
\end{tabular}

In contrast, to the SBT scheme, the received video quality performance of the test video sequences with high motion characterization performed poorly under same channel condition. The poor quality performance is due to insufficient protection against the impact of channel errors. The quality enhancement (PSNR value) as observed in the case of Soccer test video sequence from $33.52 \mathrm{~dB}$ (without $\mathrm{SBT}$ ) to $35.23 \mathrm{~dB}$ (with SBT) is a result of enhancement in the error protection level in terms of improvement in the SNR. Consequently, the performance of Akiyo and Weather characterize with low motion characterization is relatively low but perform above average acceptable PSNR value [37] [38].

\section{CONCLUSION AND Future WORK}

Video communications is gaining popularity in contrast to voice communication. However, supporting improved video quality over constrained network is challenging due to dynamic channel characteristics, network constraints. Hence, Smart Broadcast Technique (SBT) is proposed for improved quality of video applications over constrained network. SBT scheme has been discussed and analyzed in this paper as a solution to the challenge. A solution, which strategically improves the average, received video quality performance over a constrained network through intelligent distribution of the limited network resources. The experimental results evaluate the capability of the proposed SBT scheme. Overall, the scheme could be beneficial to broadcast service operators as they can maximize the average received video quality performance under a constrained network, which can lead to more viewers' satisfaction.

To further improve the proposed system performance, future work looks into more advanced mechanisms to further enhance the average video quality performance of over a constrained network.

\section{REFERENCES}

[1] G. J. Sullivan and T. Wiegand, "Video Compression - From Concepts to the H.264/AVC Standard," Proceedings of the IEEE, vol. 93, pp. 18-31, 2005.

[2] T. Stockhammer, M. M. Hannuksela, and T. Wiegand, "H.264/AVC in wireless environments," Circuits and Systems for Video Technology, IEEE Transactions on, vol. 13, pp. 657-673, 2003.

[3] S. Kumar and et al, "Error Resiliency Schemes in H.264/AVC Standard," Elsevier Journal of Visual Communication and Image Representation, Vol.17, pp. 424-450, 2006.

[4] F. Beltran, X. Gutie, and J. Melus, "Technology and market conditions toward a new competitive landscape in the wireless access market," Communications Magazine, IEEE, vol. 48, pp. 46-52, 2010.

[5] S. Ahmadi, "An overview of next-generation mobile WiMAX technology," Communications Magazine, IEEE, vol. 47, pp. 84-98, 2009.

[6] L. Hanso, "Video Compression Communications," John Wiley \& Sons, Ltd, West Sussex, England, United Kingdom, 2007.

[7] L. M. Correia, "Mobile Broadband Multimedia Networks: Techniques, Models and Tools for 4G," Academic Press, London, United Kingdom, 2006.

[8] R. Jain, S.-I. Chakchai, and A. k. Al Tamimi, "System-level modeling of IEEE $802.16 \mathrm{E}$ mobile wimax networks: Key issues," Wireless Communications, IEEE, vol. 15, pp. 73-79, 2008.

[9] Z. Han and K. Liu, "Resource Allcoation for Wireless Networks: Basics, Techniques, and Applications," Cambridge University Press, 2008.

[10] Z. Ahmad, S. Worrall, and A. Kondoz, "Unequal power allocation for scalable video transmission over WiMAX," in Multimedia and Expo, 2008 IEEE International Conference on, 2008, pp. 517-520.

[11] S. Nasir, S. Worrall, M. Mrak, and A. M. Kondoz, "An unequal error protection scheme for object based video communications," in Consumer Electronics, 2008. ISCE 2008. IEEE International Symposium on, 2008, pp. 1-4.

[12] R. arumugam, V. Subramanian and A. A. Minai, "SCRIBE: Selforganised Content and Routung in Intelligent Broadcast Environments", Military Communications Conference, 2003. MILCOM 2003.

[13] S. H. Jin, T. M. Bae, Y. M. Ro, H. Kim and M. Kim, "Intelligent Broadcast System and Services for Personalised Semantic Contents Consumption", Expert Systems with Applications 31 (2006) 164-173, Elsevier Limited.

[14] G.M. Muntean, G. Ghinea, and T.N. Sheehan, "Region of Interest based Dptive multimedia Streaming Scheme," IEEE Transactions Broadcast, Vol. 54, No. 2, June 2008.

[15] W. Song, D.W. Tjondronegoro, S.H. Wang, and M.J. Docherty, "Impact of Zooming and Enhancing Region of Interest for Optimising User Experience on Mobile Sport Videos", Proc. Intl. Conf. Multimedia, 2010.

[16] Y. Sun, I. Ahmad, and Y.Q. Zhang, "Region-based Rate Control and Bit Allocation for Wireless Video Transmission," IEEE Transactions on Multimedia Vol. 8, No. 1, February 2006.

[17] S.R. Gulliver and GG. ghinea, "A Perceptual Comaprison of Emprical and Predictive Region-of-Interest Video", IEEE Trans. Syst. Human, vol. 39, No.4, July 2009.

[18] D. Fleet and Y. Wiess, "Optical Flow Estimation in Paragios. ," Handbook of Mathematical Models in Computer Vision, Springer, 2006.

[19] Jianbo and et al, "Good features to track " IEEE Computer Society Conference on Computer Vision and Pattern Recognition, 1994.

[20] I. E. G. Richardson, "H.264 and MPEG-4 Video Compression," John Wiley and Sons Limited. West Sussex, England, 20032003.

[21] G. Nur, S. Dogan, H. Kodikara Arachchi and A.M. Kondoz, "Impact of Depth Map Spatial Resolution on 3D Video Quality and Depth Perception", Processings of the 4th IEEE 3DTV Conference, Tampere, Finland, 7-9 June 201.

[22] T. Wiegand, G. J. Sullivan, G. Bjontegaard, and A. Luthra, "Overview of the H.264/AVC video coding standard," Circuits and Systems for Video Technology, IEEE Transactions on, vol. 13, pp. 560-576, 2003. 
[23] R.G. Gallager, "Energy Limited Channels: Coding, Multiacces and Spread Spectrum", Tech. REP. MIT. LIDS-P-1714, Novermber 1987.

[24] O. Oyman, J. Foerster, T. Yong-joo, and L. Seong-Choon, "Toward enhanced mobile video services over WiMAX and LTE [WiMAX/LTE Update]," Communications Magazine, IEEE, vol. 48, pp. 68-76, 2010.

[25] L. Ke-Ying, Y. Jar-Ferr, and S. Ming-Ting, "Rate-Distortion Cost Estimation for H.264/AVC," Circuits and Systems for Video Technology, IEEE Transactions on, vol. 20, pp. 38-49, 2010.

[26] W. Yao, W. Zhenyu, and J. M. Boyce, "Modeling of transmission-lossinduced distortion in decoded video," Circuits and Systems for Video Technology, IEEE Transactions on, vol. 16, pp. 716-732, 2006.

[27] A. M. Tourapis, "Joint Video Team (JVT) of ISO/IEC MPEG \& ITU-T VCEG (ISO/IEC JTC1/SC29/WG11 and ITU-T SG16 Q.6)," Geneva, http://iphome.hhi.de/suehring/tml/, February 2009.

[28] S. Wenger, "H.264/AVC over IP," Circuits and Systems for Video Technology, IEEE Transactions on, vol. 13, pp. 645-656, 2003.

[29] S. Wenger, M. Hannuksela, T. Stockhammer, M. Westerland, and D. Singer, "RTP Payload Format for H.264 Video," IETF, RFC 3984, February 2005.

[30] J. Ostermann, J. Bormans, P. List, D. Marpe, M. Narroschke, F. Pereira, T. Stockhammer, and T. Wedi, "Video coding with H.264/AVC: tools, performance, and complexity," Circuits and Systems Magazine, IEEE, vol. 4, pp. 7-28, 2004.

[31] S. Jung, "Effect of Robust Header Compression (ROHC) and Packet
Aggregation on Multi-hop Wireless Mesh Networks," IEEE 6th International Conference on computer and Information Technology (CIT'06), 2006.

[32] S. C. H. Schulzrinne, R. Frederick, V. Jacobson, "RTP: A Transport Protocol for Real-Time Applications, RFC 3550 Internet-Draft," IETF Network Working Group, July 2003.

[33] EU-1ST-FP6-Project, "Scalable Ultra-fast and Interoperable Interactive Television (SUIT)," http://suit.av.it.pt/, 2006.

[34] A.A. Atayero, O.I. Sheluhim, Y.A. Ivanov and A.A. Alatishe, "Estimation of the Visual Quality of Video Streaming Under Desynchronisation Conditions", International Journal of Advanced Computer Science and Aplications, Vol. 2, No. 12, 2011.

[35] M. Vranjes, S. Rimac-Drlje, and K. Grgic, "Locally averaged PSNR as a simple objective Video Quality Metric," in ELMAR, 2008. 50th International Symposium, 2008, pp. 17-20.

[36] A. M. Kondoz, "Visual Media Coding and Transmission," John Wiley \& Sons Ltd, United Kingdom, 2009.

[37] A.A. Atayero, O.I. Sheluhim, Y.A. Ivanov and J.O. Iruemi, "Wideband Wireless Access Systems Interference Robustness: Its Effect on Quality of Video Streaming", International Journal of Advanced Computer Science and Aplications, Vol. 3, No. 1, 2012.

[38] T. Zinner, O. Abboud, O. Hohlfeld, and P. Tran-Gia, "Toward QoE Management for Scalable Video Streaming," Proc. 21th ITC Specialist Seminar Multimedia Appl. Traffic, performance QoE, 2010. 